\title{
The B-nor-methylene colchicinoid PT-100 selectively induces apoptosis in multidrug resistant human cancer cells via the intrinsic pathway in a caspase-independent manner
}

\author{
Andreas Stein ${ }^{\ddagger a}$, Persefoni Hilken née Thomopoulou ${ }^{\ddagger a}$, Corazon Frias ${ }^{b}$, Sina M. Hopff ${ }^{b}$, \\ Paloma Varela ${ }^{c}$,Nicola Wilke ${ }^{b}$,Arul Mariappan ${ }^{d}$, Jörg-Martin Neudörfl ${ }^{a}$, Alexey Yu. \\ Fedorove, Jay Gopalakrishnan ${ }^{\text {, Benoit Gigant }}{ }^{c}$, Aram Prokop*b.f, and Hans-Günther \\ Schmalz*a
}

\section{AUTHOR ADDRESS}

a Department of Chemistry, University of Cologne, 50939 Cologne, Germany

${ }^{\mathrm{b}}$ Department of Paediatric Oncology, Children's Hospital Cologne, 50735 Cologne, Germany

c Université Paris-Saclay, CEA, CNRS, Institute for Integrative Biology of the Cell (I2BC), 91198, Gif-sur-Yvette cedex, France

${ }^{\mathrm{d}}$ Laboratory for Centrosome and Cytoskeleton Biology, Institute of Human Genetics, Heinrich-Heine-University, 40225 Düsseldorf, Germany

${ }^{\text {e }}$ Department of Organic Chemistry, N.I. Lobachevsky State University of Nizhny Novgorod, 603950 Nizhny Novgorod, Russian Federation

${ }_{\mathrm{f}}^{\mathrm{f}}$ Department of Pediatric Hematology/Oncology, Helios Clinic Schwerin, 19055 Schwerin, Germany; and MSH Medical School Hamburg, Am Kaiserkai 1, 20457 Hamburg, Germany

\section{Corresponding Author}

* A. P.: Tel.: +49-385-520-6396; E-Mail: Aram.Prokop@helios-gesundheit.de

*H.-G. S.: Tel.: +49-221-470-3063; E-Mail: schmalz@uni-koeln.de 


\section{Table of Contents}

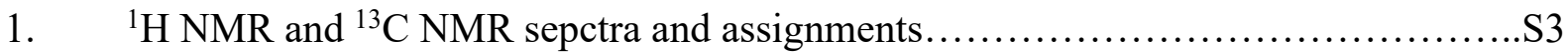

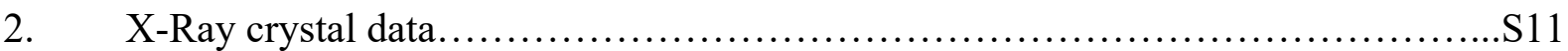




\section{${ }^{1} \mathrm{H}$ NMR and ${ }^{13} \mathrm{C}$ NMR sepctra and assignments}

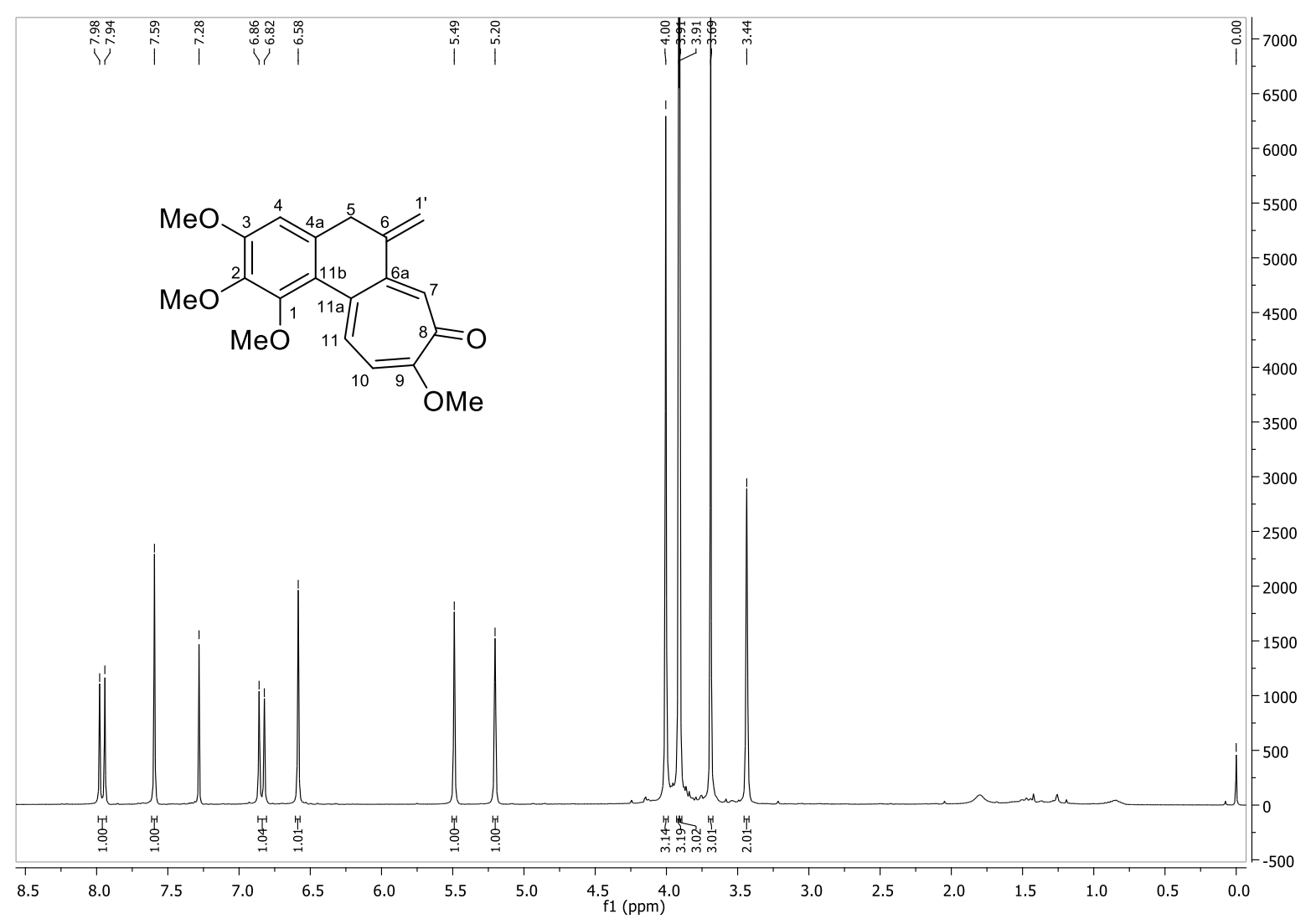

Figure S1: ${ }^{1} \mathrm{H}$ NMR spectrum of compound $7\left(\mathrm{CDCl}_{3}, 400 \mathrm{MHz}\right)$

$\delta[\mathrm{ppm}]=3.44(\mathrm{~s}, 2 \mathrm{H}, \mathrm{H}-5), 3.69(\mathrm{~s}, 3 \mathrm{H}, \mathrm{OMe}), 3.91(\mathrm{~s}, 6 \mathrm{H}, 2 \mathrm{xOMe}), 4.00(\mathrm{~s}, 3 \mathrm{H}, \mathrm{OMe}), 5.20$ (s, 1H, H-1'), 5.49 (s, 1H, H-1'), 6.58 (s, 1H, H-4), 6.84 (d, $\left.{ }^{3} J_{\mathrm{H}, \mathrm{H}}=10.9 \mathrm{~Hz}, 1 \mathrm{H}, \mathrm{H}-10\right), 7.59$ (s, $1 \mathrm{H}, \mathrm{H}-7), 7.96\left(\mathrm{~d},{ }^{3} J_{\mathrm{H}, \mathrm{H}}=10.9 \mathrm{~Hz}, 1 \mathrm{H}, \mathrm{H}-11\right)$. 


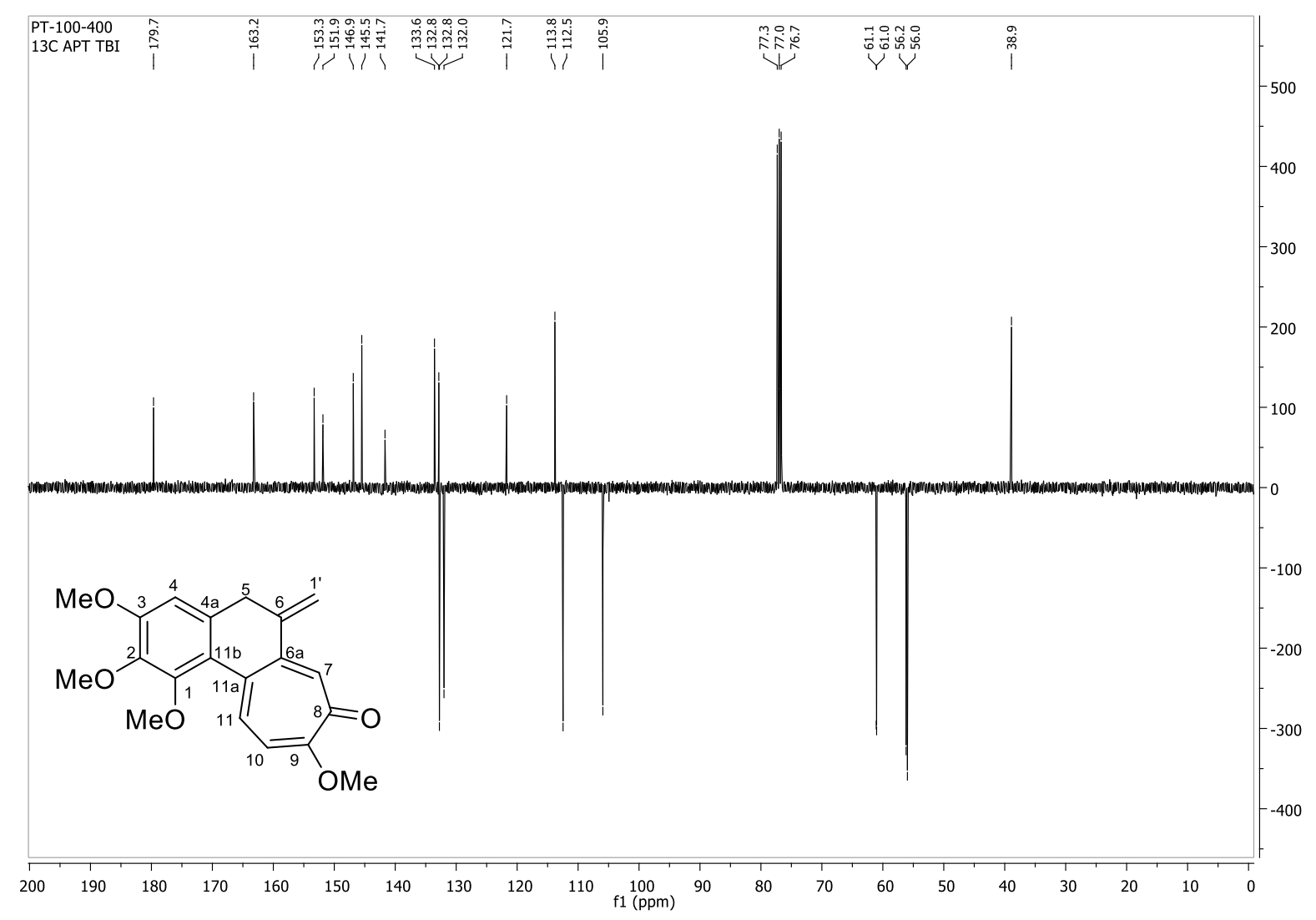

Figure S2: ${ }^{13} \mathrm{C}$ NMR spectrum of compound $7\left(\mathrm{CDCl}_{3}, 100 \mathrm{MHz}\right)$

$\delta[\mathrm{ppm}]=38.9(\mathrm{C}-5), 56.0(\mathrm{OMe}), 56.2(\mathrm{OMe}), 61.0(\mathrm{OMe}), 61.1(\mathrm{OMe}), 105.9(\mathrm{C}-4), 112.5$ (C-10), 113.8 (C-1'), 121.7 (C-11b), 132.0 (C-11), 132.7 (C-7), 132.8 (C-11a), 133.6 (C-4a), 141.7 (C-3), 145.5 (C-6), 146.9 (C-6a), 151.9 (C-1), 153.3 (C-2), 163.2 (C-9), 179.7 (C-8). 


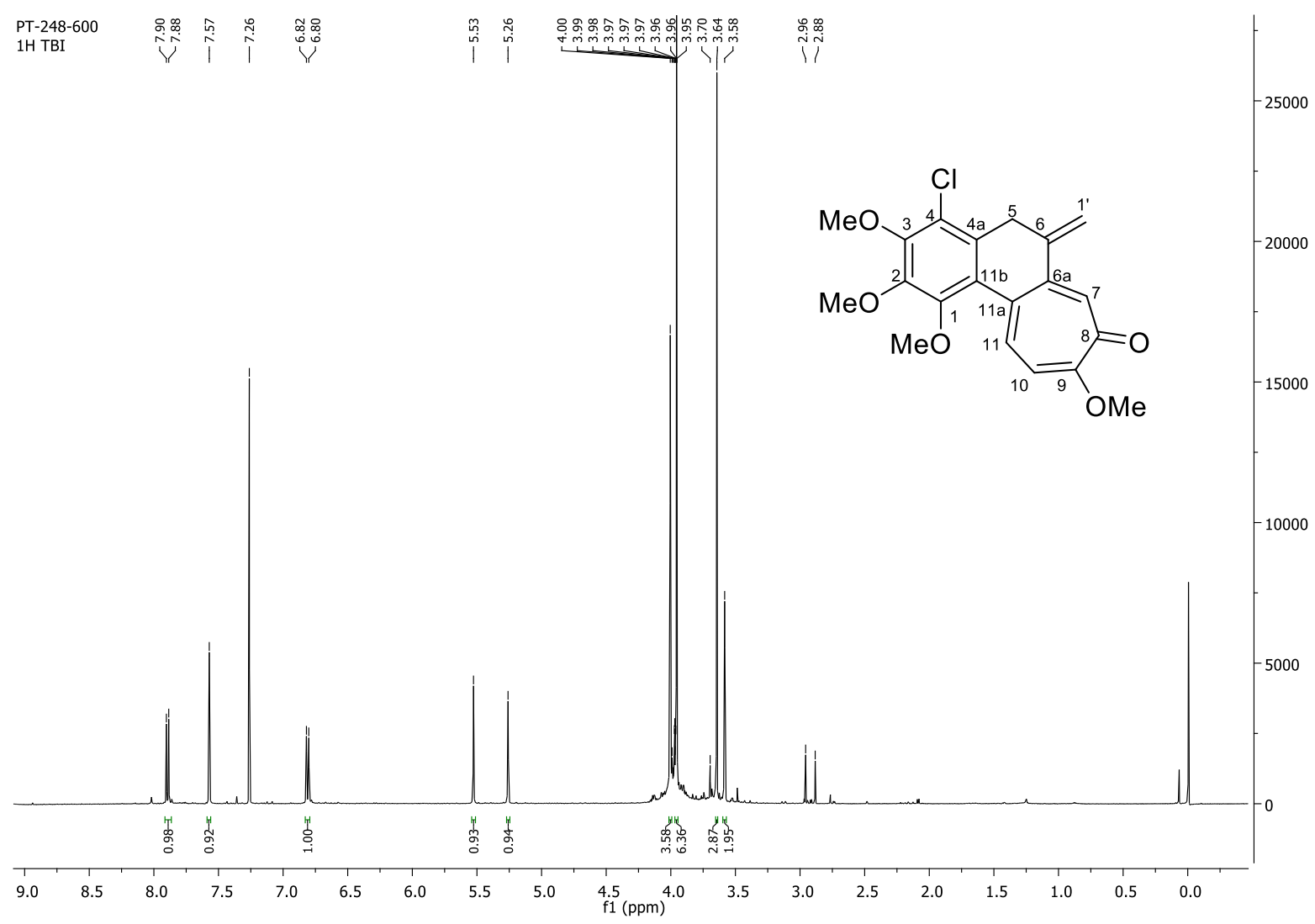

Figure S3: ${ }^{1} \mathrm{H}$ NMR spectrum of compound $9\left(\mathrm{CDCl}_{3}, 600 \mathrm{MHz}\right)$

$\delta[\mathrm{ppm}]=3.58(\mathrm{~s}, 2 \mathrm{H}, \mathrm{H}-5), 3.64(\mathrm{~s}, 3 \mathrm{H}, \mathrm{OMe}), 3.95(\mathrm{~s}, 3 \mathrm{H}, \mathrm{OMe}), 3.96(\mathrm{~s}, 3 \mathrm{H}, \mathrm{OMe}), 4.00(\mathrm{~s}$, $3 \mathrm{H}, \mathrm{OMe}), 5.26$ (s, 1H, H-1'), 5.53 (s, 1H, H-1'), 6.81 (d, $\left.{ }^{3} J_{\mathrm{H}, \mathrm{H}}=10.9 \mathrm{~Hz}, 1 \mathrm{H}, \mathrm{H}-10\right), 7.57$ (s, $1 \mathrm{H}, \mathrm{H}-7), 7.90\left(\mathrm{~d},{ }^{3} J_{\mathrm{H}, \mathrm{H}}=10.9 \mathrm{~Hz}, 1 \mathrm{H}, \mathrm{H}-11\right)$. 


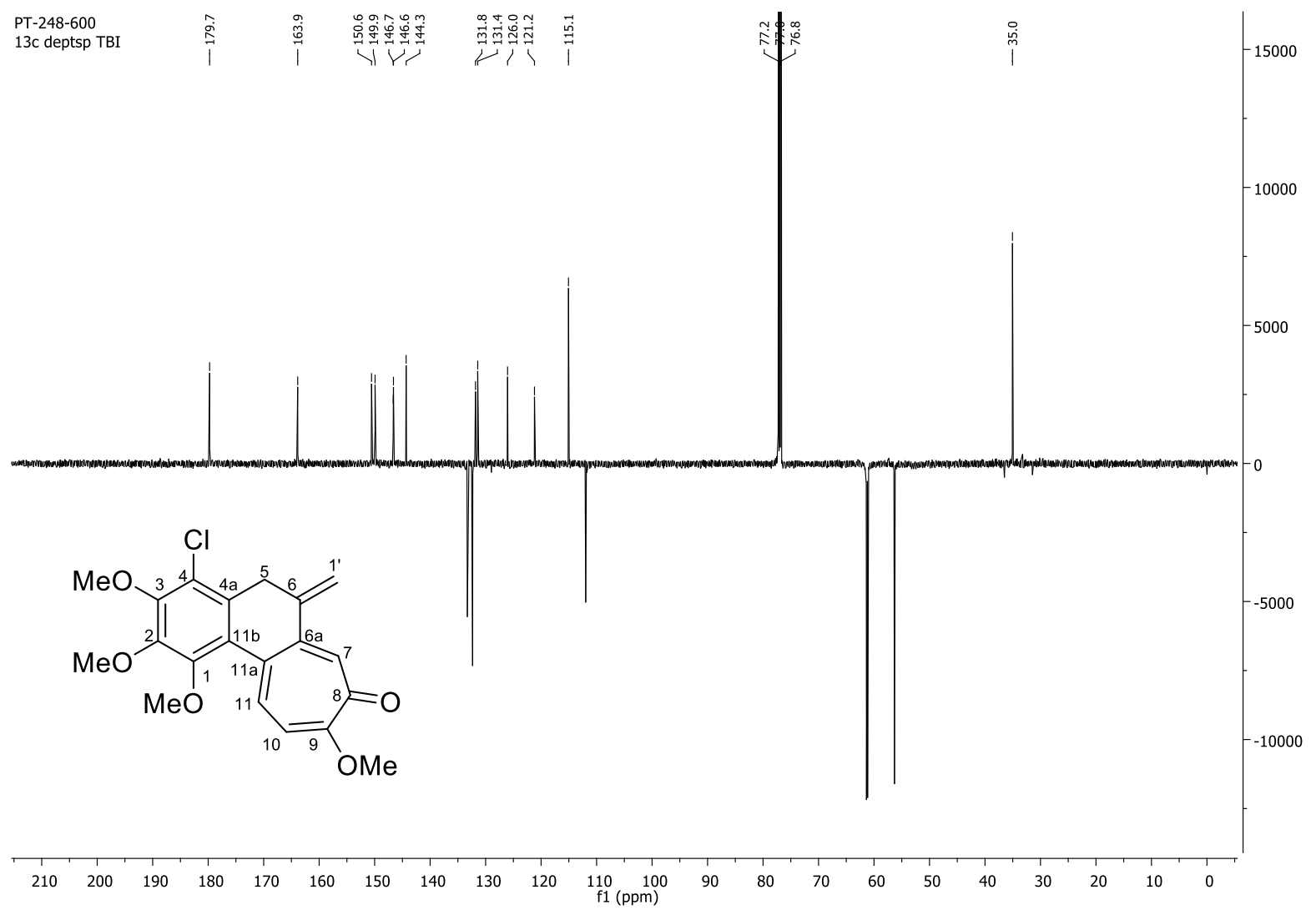

Figure S4: ${ }^{13} \mathrm{C}$ NMR spectrum of compound $9\left(\mathrm{CDCl}_{3}, 150 \mathrm{MHz}\right)$

$\delta[\mathrm{ppm}]=38.1(\mathrm{C}-5), 56.3(\mathrm{OMe}), 60.2(\mathrm{OMe}), 61.3(\mathrm{OMe}), 61.4(\mathrm{OMe}), 112.0(\mathrm{C}-10), 115.1$ (C-1'), 121.2 (C-4), 126.0 (C-11b), 131.4 (C-11a), 131.8 (C-4a), 132.4 (C-7), 133.3 (C-11), 144.3 (C-6), 146.6 (C-3), 146.7 (C-6a), 149.9 (C-1), 150.6 (C-2), 163.9 (C-9), 179.7 (C-8). 


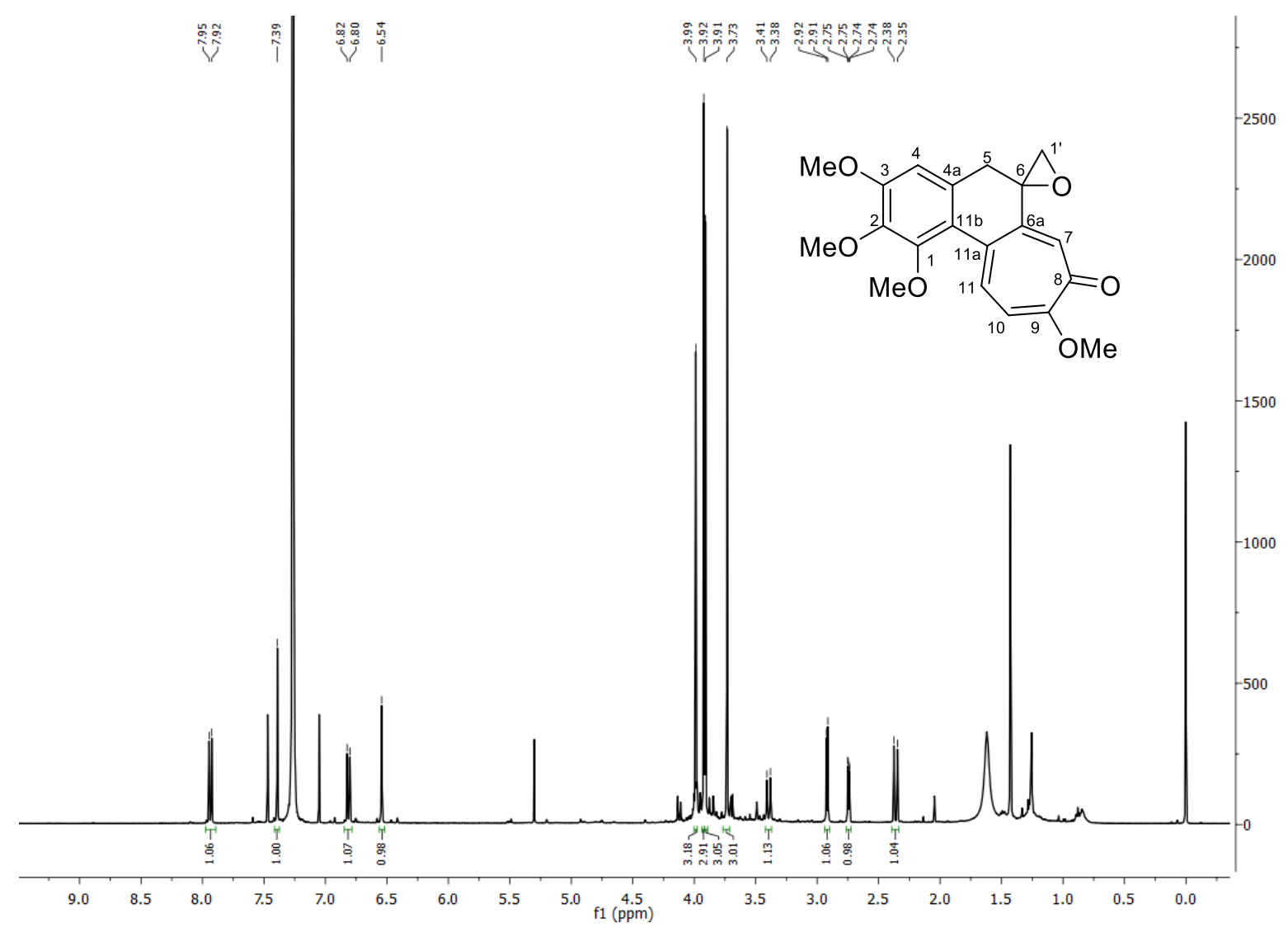

Figure S5: ${ }^{1} \mathrm{H}$ NMR spectrum of compound $\mathrm{rac}-\mathbf{1 0}\left(\mathrm{CDCl}_{3}, 500 \mathrm{MHz}\right)$

$\delta[\mathrm{ppm}]=2.36\left(\mathrm{~d}, J_{\mathrm{H}, \mathrm{H}}=14.6 \mathrm{~Hz}, 1 \mathrm{H}, \mathrm{H}-5 \mathrm{a}\right), 2.74\left(\mathrm{dd}, J_{\mathrm{H}, \mathrm{H}}=5.5,1.7 \mathrm{~Hz} ; 1 \mathrm{H}, \mathrm{H}-1\right.$ ' a $), 2.92(\mathrm{~d}$, $J_{\mathrm{H}, \mathrm{H}}=5.5 \mathrm{~Hz}, 1 \mathrm{H}, \mathrm{H}-1$ 'b), 3.39 (dt, $\left.J_{\mathrm{H}, \mathrm{H}}=14.4,1.2 \mathrm{~Hz} ; 1 \mathrm{H}, \mathrm{H}-5 \mathrm{~b}\right), 3.73$ (s, 3H, OMe), 3.91 (s, $3 \mathrm{H}, \mathrm{OMe}), 3.92$ (s, 3H, OMe), 3.99 (s, 3H, OMe), $6.54(\mathrm{~s}, 1 \mathrm{H}, \mathrm{H}-4), 6.81\left(\mathrm{~d},{ }^{3} J_{\mathrm{H}, \mathrm{H}}=11.1 \mathrm{~Hz}\right.$, $1 \mathrm{H}, \mathrm{H}-10), 7.39$ (s, 1H, H-7), $7.94\left(\mathrm{~d},{ }^{3} J_{\mathrm{H}, \mathrm{H}}=10.9 \mathrm{~Hz}, 1 \mathrm{H}, \mathrm{H}-11\right)$. 


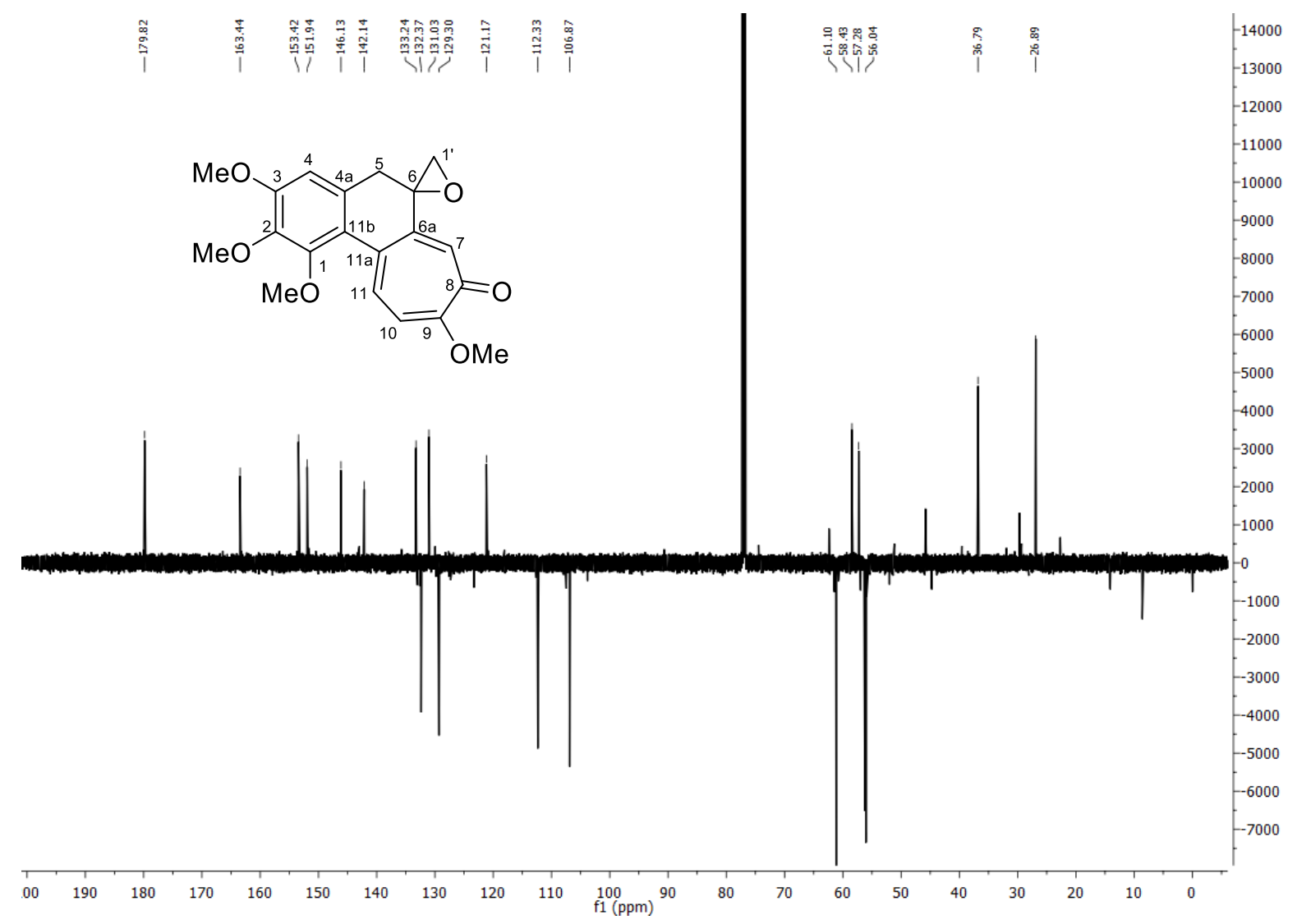

Figure S6: ${ }^{13} \mathrm{C}$ NMR spectrum of compound $\mathrm{rac}-\mathbf{1 0}\left(\mathrm{CDCl}_{3}, 125 \mathrm{MHz}\right)$

$\delta[\mathrm{ppm}]=36.79(\mathrm{C}-5), 56.04(\mathrm{OMe}), 56.21(\mathrm{OMe}), 57.28\left(\mathrm{C}-1^{\prime}\right), 58.43(\mathrm{C}-6), 61.10(2 \times \mathrm{OMe})$, 106.87 (C-4), 112.33 (C-10), 121.17 (C-11b), 129.30 (C-7), 131.03 (C-4a), 132.37 (C-11), 133.24 (C-11a), 142.14 (C-2), 146.13 (C-6a), 151.94 (C-1), 153.42 (C-3), 163.44 (C-9), 179.82 (C-8). 


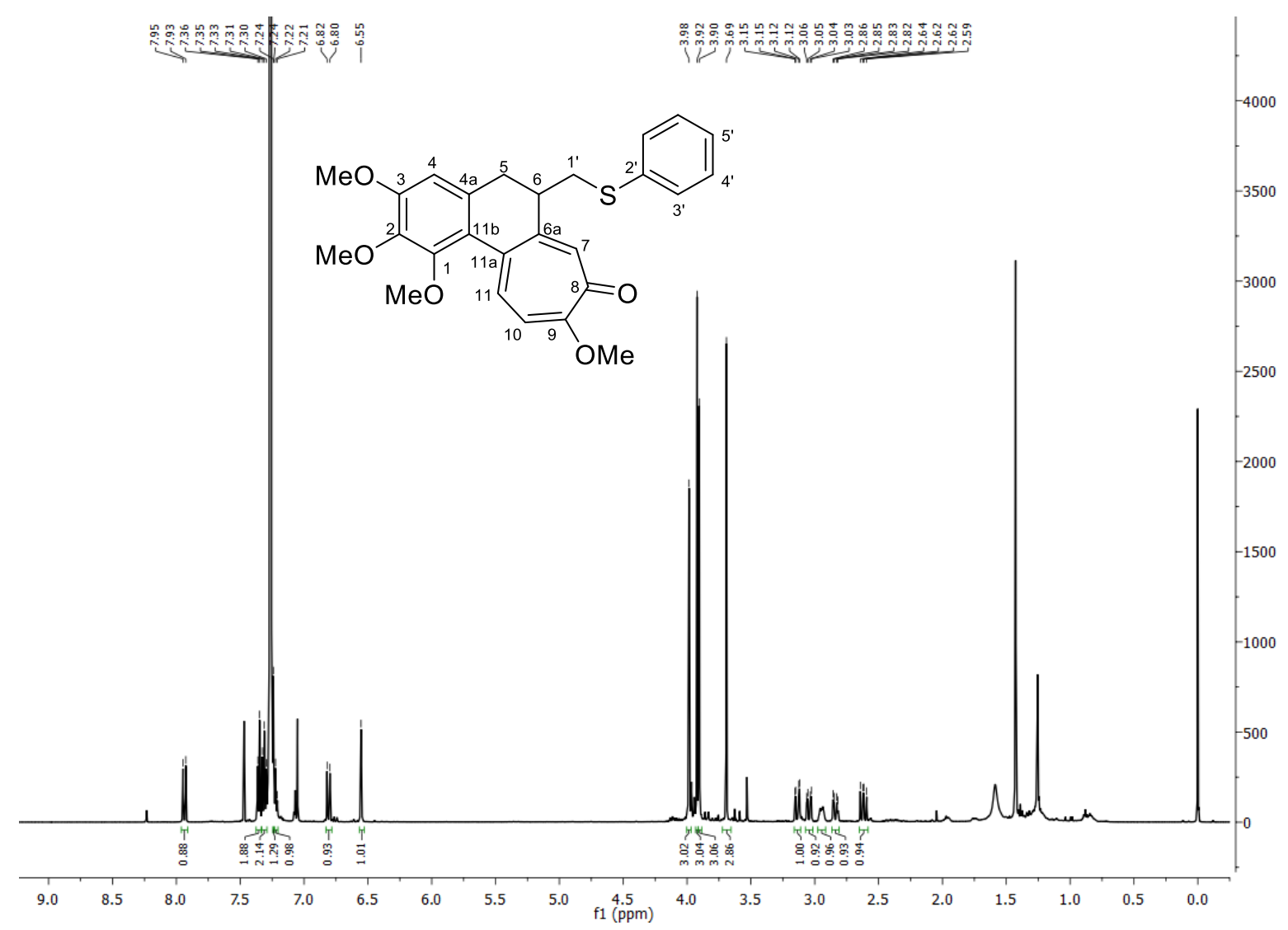

Figure S7: ${ }^{1} \mathrm{H}$ NMR spectrum of compound $\mathrm{rac}-11\left(\mathrm{CDCl}_{3}, 500 \mathrm{MHz}\right)$

$\delta[\mathrm{ppm}]=2.62\left(\mathrm{dd}, J_{\mathrm{H}, \mathrm{H}}=13.7 \mathrm{~Hz}, 10.9 \mathrm{~Hz} ; 1 \mathrm{H}, \mathrm{H}-1\right.$ ' a), $2.84\left(\mathrm{dd}, J_{\mathrm{H}, \mathrm{H}}=15.0 \mathrm{~Hz}, 4.3 \mathrm{~Hz} ; 1 \mathrm{H}\right.$, H-5a), 2.92-297 (m, 1H, H-6), 3.05 (dd, $J_{\mathrm{H}, \mathrm{H}}=13.7 \mathrm{~Hz}, 4.7 \mathrm{~Hz} ; 1 \mathrm{H}, \mathrm{H}-1$ ’b), 3.14 (dd, $J_{\mathrm{H}, \mathrm{H}}=15.1 \mathrm{~Hz}, 2.2 \mathrm{~Hz}$; 1H, H-5b), 3.69 (s, 3H, OMe), 3.90 (s, 3H, OMe), 3.92 (s, 3H, OMe), $9.98(\mathrm{~s}, 3 \mathrm{H}, \mathrm{OMe}), 6.55(\mathrm{~s}, 1 \mathrm{H}, \mathrm{H}-4), 6.81\left(\mathrm{~d},{ }^{3} J_{\mathrm{H}, \mathrm{H}}=11.1 \mathrm{~Hz}, 1 \mathrm{H}, \mathrm{H}-10\right), 7.22\left(\mathrm{t},{ }^{3} J_{\mathrm{H}, \mathrm{H}}=7.2\right.$ $\left.\mathrm{Hz}, 1 \mathrm{H}, \mathrm{H}-5^{\prime}\right), 7.24$ (s, 1H, H-7), 7.31 (t, $\left.{ }^{3} J_{\mathrm{H}, \mathrm{H}}=8.2 \mathrm{~Hz}, 2 \mathrm{H}, \mathrm{H}-4^{\prime}\right), 7.36\left(\mathrm{~d},{ }^{3} J_{\mathrm{H}, \mathrm{H}}=7.1 \mathrm{~Hz}, 2 \mathrm{H}\right.$, H-3'), $7.94\left(\mathrm{~d},{ }^{3} J_{\mathrm{H}, \mathrm{H}}=11.0 \mathrm{~Hz}, 1 \mathrm{H}, \mathrm{H}-11\right)$. 


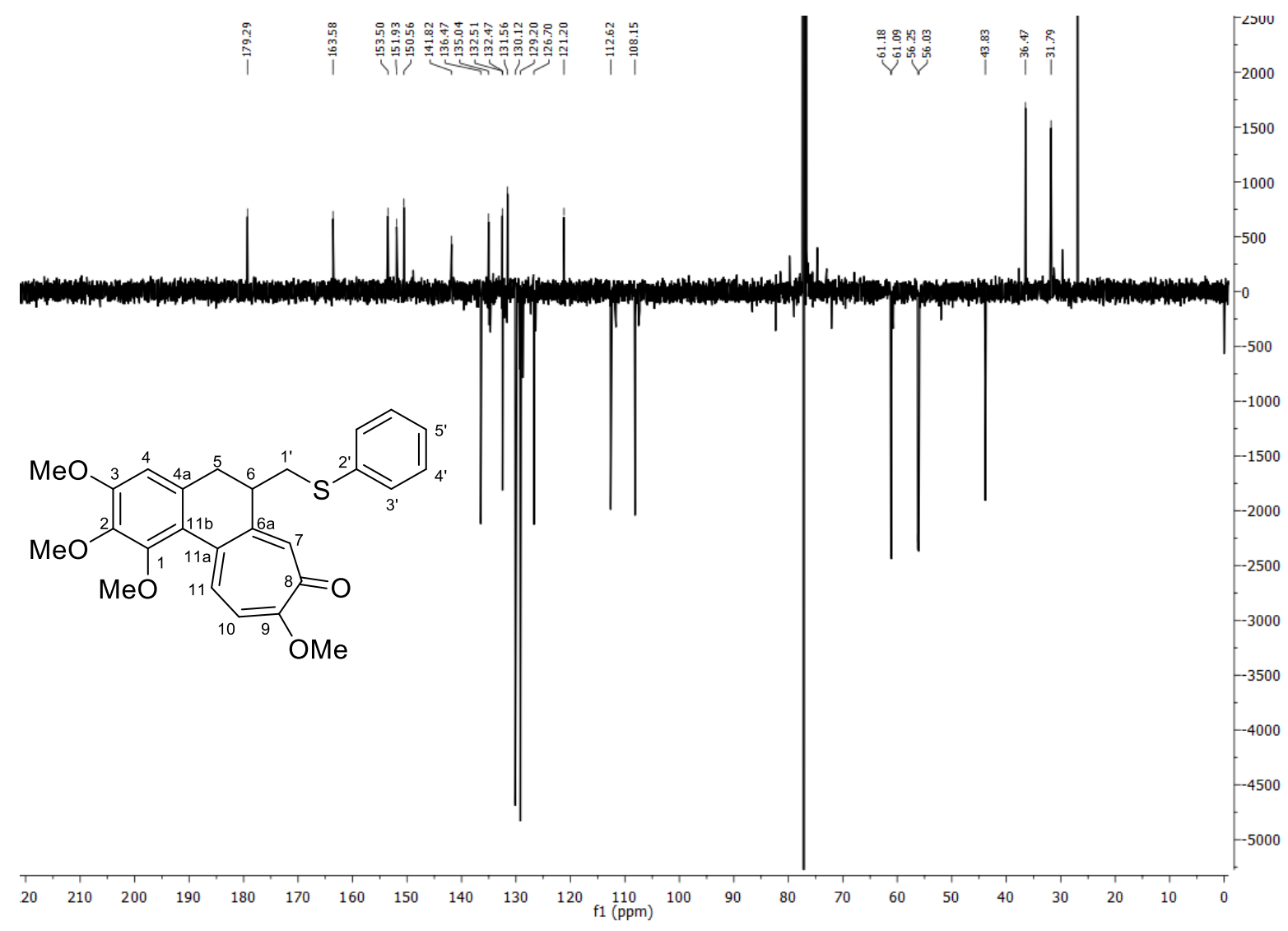

Figure S8: ${ }^{13} \mathrm{C}$ NMR spectrum of compound $\mathrm{rac}-\mathbf{1 1}\left(\mathrm{CDCl}_{3}, 125 \mathrm{MHz}\right)$

$\delta$ [ppm] $=31.79(\mathrm{C}-5), 36.47(\mathrm{C}-1$ ') $, 43.83(\mathrm{C}-6), 56.03(\mathrm{OMe}), 56.25(\mathrm{OMe}), 61.09(\mathrm{OMe})$, 61.18 (OMe), 108.15 (C-4), 112.62 (C-10), 121.20 (C-11b), 126.70 (C-5'), 129.20 (C-4'), 130.12 (C-3'), 131.56 (C-4a), 132.47 (C-11), 132.51 (C-11a), 135.04 (C-2'), 136.47 (C-7), 141.82 (C-2), 150.56 (C-6a), 151.93 (C-1), 153.50 (C-3), 163.58 (C-9), 179.29 (C-8). 


\section{X-Ray crystallographic data}

\section{Compound 7}

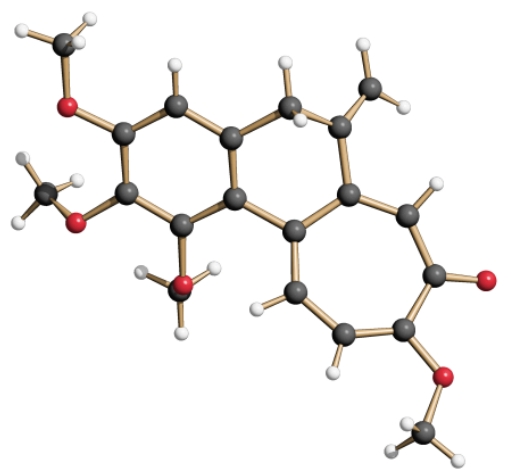

Figure S9: Structure of compound 7 in the crystalline state.

Table S1: Crystal data and structure refinement for 7.

Identification code

pt100

Empirical formula

C20 H20 O5

Formula weight

340.36

Temperature

100(2) K

Wavelength

$0.71073 \AA$

Crystal system, Space group

Triclinic, P-1

Unit cell dimensions

$\mathrm{a}=7.6466(11) \AA$

$\alpha=71.786(9)^{\circ}$

$\mathrm{b}=9.1687(14) \AA$

$\beta=83.343(7)^{\circ}$

$\mathrm{c}=12.588(2) \AA$

$\gamma=85.612(9)^{\circ}$

Volume

831.9(2) $\AA^{3}$

Z

Density (calculated)

Absorption coefficient

$1.359 \mathrm{Mg} / \mathrm{m}^{3}$

$\mathrm{F}(000)$

Crystal size

Theta range for data collection

$0.097 \mathrm{~mm}^{-1}$

360

Index ranges

Reflections collected

$0.200 \times 0.150 \times 0.030 \mathrm{~mm}^{3}$

1.711 to $24.995^{\circ}$.

$-8<=\mathrm{h}<=9,-8<=\mathrm{k}<=10,-14<=\mathrm{l}<=14$

Independent reflections

Completeness to theta $=25.242^{\circ}$

4479

$2910[\mathrm{R}($ int $)=0.0539]$

$96.7 \%$

Absorption correction

None

Refinement method

Full-matrix least-squares on $\mathrm{F}^{2}$

Data / restraints / parameters

2910 / 0 / 238

Goodness-of-fit on $\mathrm{F}^{2}$

0.912

Final $\mathrm{R}$ indices $[\mathrm{I}>2 \operatorname{sigma}(\mathrm{I})]$

$\mathrm{R} 1=0.0560, \mathrm{wR} 2=0.1147$

$\mathrm{R}$ indices (all data)

$\mathrm{R} 1=0.1534, \mathrm{wR} 2=0.1653$

Extinction coefficient

$\mathrm{n} / \mathrm{a}$

Largest diff. peak and hole

0.262 and -0.303 e. $\AA^{-3}$ 


\section{Compound rac-10}

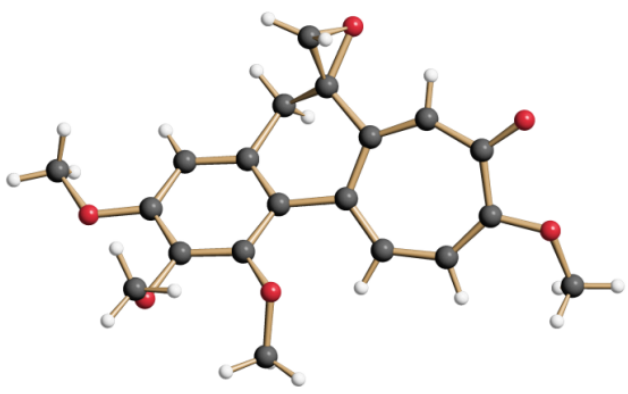

Figure S10: Structure of compound $r a c-10$ in the crystalline state

Table S2: Crystal data and structure refinement for compound rac-10.

Identification code

Empirical formula

Moiety formula

Formula weight

Temperature

Wavelength

Crystal system

Space group

Unit cell dimensions

Volume

Z

Density (calculated)

Absorption coefficient

$\mathrm{F}(000)$

Crystal size

Theta range for data collection

Index ranges

Reflections collected

Independent reflections

Completeness to theta $=66.865^{\circ}$

Absorption correction

Max. and min. transmission

Refinement method

Data / restraints / parameters

Goodness-of-fit on $\mathrm{F}^{2}$

Final $\mathrm{R}$ indices $[\mathrm{I}>2 \operatorname{sigma}(\mathrm{I})]$

$\mathrm{R}$ indices (all data)

Extinction coefficient

Largest diff. peak and hole asda042

C20 H20 O6

$\mathrm{C} 20 \mathrm{H} 20 \mathrm{O} 6$

356.36

100(2) K

$1.54178 \AA$

Triclinic

P-1

$$
\begin{array}{ll}
\mathrm{a}=9.417(14) \AA & \alpha=98.68(5)^{\circ} . \\
\mathrm{b}=10.508(4) \AA & \beta=104.86(10)^{\circ} . \\
\mathrm{c}=18.010(12) \AA & \gamma=92.55(7)^{\circ} .
\end{array}
$$

1696(3) $\AA^{3}$

4

$1.395 \mathrm{Mg} / \mathrm{m}^{3}$

$0.857 \mathrm{~mm}^{-1}$

752

$0.100 \times 0.015 \times 0.015 \mathrm{~mm}^{3}$

2.575 to $66.865^{\circ}$.

$-11<=\mathrm{h}<=11,-12<=\mathrm{k}<=11,-21<=\mathrm{l}<=21$

30207

$5988[\mathrm{R}(\mathrm{int})=0.0853]$

$99.1 \%$

Semi-empirical from equivalents

0.7528 and 0.5643

Full-matrix least-squares on $\mathrm{F}^{2}$

5988 / 0 / 477

1.084

$\mathrm{R} 1=0.0598, \mathrm{wR} 2=0.1067$

$\mathrm{R} 1=0.0887, \mathrm{wR} 2=0.1160$

$\mathrm{n} / \mathrm{a}$

0.278 and -0.272 e. $\AA^{-3}$ 
$\mathrm{T}_{2}$ R Complex with PT-100 (7)

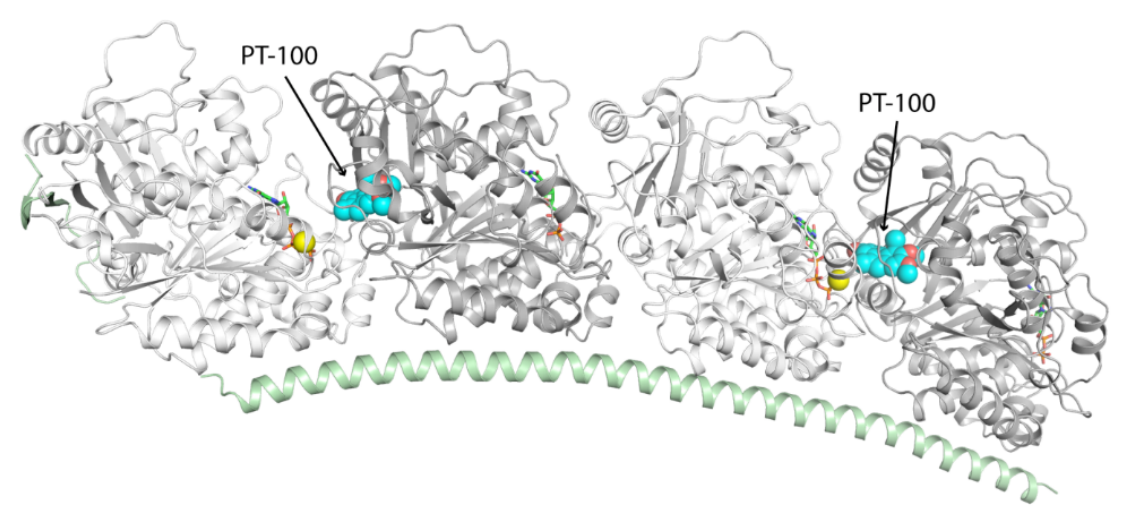

Figure S11: Structure of complex T2R:PT-100 (7) in the crystalline state.

Table S3: Crystal data and structure refinement for complex T2R:PT-100.

Data collection $^{\text {a }}$

Space group

Cell dimensions

$$
\mathrm{P} 2{ }_{1} 2{ }_{1}{ }_{1}
$$

$\mathrm{a}=65.12 \AA$

$\alpha=90.0^{\circ}$

$\mathrm{b}=128.30 \AA$

$\beta=90.0^{\circ}$

$\mathrm{c}=252.72 \AA$

$\gamma=90^{\circ}$

Resolution $(\AA)$

$126-2.12(2.42-2.12)$

Anisotropy resolution limits ${ }^{\mathrm{b}}$

$3.16,2.06,2.65$

$\mathrm{R}_{\text {meas }}$

$0.076(1.151)$

I / $\sigma \mathrm{I}$

$15.1(1.8)$

$\mathrm{CC}_{1 / 2}$

$0.999(0.627)$

Completeness (spherical)

$0.520(0.081)$

$0.940(0.736)$

Multiplicity

$6.5(6.0)$

\section{Refinement}

Resolution $(\AA)$

$126-2.12$

No. reflections

62,794

$\mathrm{R}_{\text {work }} / \mathrm{R}_{\text {free }}$

$0.192 / 0.226$

No. atoms

Protein

14,512

Ligand

207

Solvent

117

B factors

Protein

66.8

Ligand

62.8

Solvent

40.5

Coordinate error $(\AA)$

0.34

R.m.s.d.

Bond lengths ( $\AA$ )

0.008

Bond angles $\left({ }^{\circ}\right)$

0.93

Ramachandran (\%)

Favored region

97.69

Allowed region

1.98

Outliers

0.33

${ }^{a}$ Data were collected on a single crystal. Values in parentheses are for the highest resolution shell. ${ }^{\mathrm{b}}$ Determined by STARANISO using local mean I / $\sigma(\mathrm{I})=1.2$. 